\title{
Evaluation of the Acridine Orange Fluorescence Technique and the Indirect Fluorescent Antibody as Diagnostic Tests for Tropical Theileriosis
}

\author{
Omer, O. H. ${ }^{1}{ }^{*}$, Mahmoud, O.M. ${ }^{2}$ and Al-Sadrani, A.A. ${ }^{2}$ \\ 1. College of Pharmacy, Qassim University, PO Box 6800 Buraidah 51452, Saudi Arabia \\ 2. College of Agriculture and Veterinary Medicine, Qassim University, Buraidah 51452, Saudi Arabia \\ Tel.: +966-6380-0050; fax: +966-6380-2268 \\ *Corresponding author email: osamaom@hotmail.com
}

Received: 11-01-2011, Accepted: 28-01-2011, Published Online: 04-06-2011

\begin{abstract}
This study was carried out to evaluate the use of acridine orange fluorescence technique on blood slides as a rapid diagnostic test for tropical theileriosis in comparison with the Giemsa-stained thin blood film technique. Also the indirect fluorescent antibody test has been employed for the serodiagnosis of tropical theileriosis. The study was carried out on 62 young and 48 adult Friesian cattle suffering from clinical tropical theileriosis in Qassim Region, Central Saudi Arabia, during the period from August 2006 to July 2008. For control, blood samples were also obtained from 25 young and 25 adult, clinically healthy, Friesian cattle, selected at random from different dairy farms in Qassim Region. Thin blood films were fixed with methanol and stained with Giemsa and acridine orange and were examined by two independent microbiologists. There was $100 \%$ correlation in the interpretation of slides stained with Giemsa and acridine orange both with respect to positivity and negativity, between the two microbiologists. It is concluded that if facilities are available acridine orange is a valuable alternative for screening tropical theileriosis. The method may also have potential value in the diagnosis of Theileria parva, which causes East Coast fever, and also other Theileria species. Results of the present study also showed that IFA test was not found sufficiently sensitive and specific as has been reported earlier.
\end{abstract}

Key words: Theileria annulata; Cattle-protozoa; Diagnosis; Acridine orange; IFA.

\section{I ntroduction}

Tropical theileriosis or Mediterranean Coast fever is a disease of cattle caused by the protozoan parasite Theileria annulata (Dschunkowsky and Luhs, 1904) and transmitted by species of ixodid ticks of the genus Hyalomma, principally Hyalomma anatolicum anatolicum (Robinson, 1982). Domestic cattle and the water buffalo (Bubalus bubalis) are susceptible, although the latter is apparently more resistant than cattle (Dhar et al., 1973). The exotic breeds and cross-bred cattle are particularly susceptible with mortality rates of $40-60 \%$ (Brown, 1990) reaching up to $80 \%$ in some areas (Gill et al., 1977; Hashemi-Fesharki, 1991).

Theileria annulata is known to occur in northern Africa, along the valley of the River Nile to at least the $13^{\text {th }}$ parallel in the Sudan, Eritrea, the Middle and Near East, Southern Europe, the Indian subcontinent, Central Asia and the Far East (Purnell, 1978; Uilenberg, 1981).
An accurate diagnosis is a prerequisite for an effective disease management of tropical theileriosis. Diagnosis of tropical theileriosis is based on clinical signs, knowledge of disease and vector distribution and examination of Giemsa-stained blood and lymph node smears (OIE, 2008).

Acridine orange (AO), a fluorescent dye, binds with nucleic acids and fluoresces. The fluorescence can easily be observed in contrast to the dark background (Kong and Chung, 1995). The acridine orange fluorescence technique has been used extensively for diagnosis of malaria (Hänscheid, 1999) and showed a good diagnostic performance when compared with Giemsa's or other staining solutions (Keiser et al., 2002).

The indirect fluorescent antibody (IFA) test has been widely used for serodiagnosis of theileriosis in cattle (Mehlhorn et al., 1994). It is sensitive and requires little standardization (OIE, 2008). However, the major disadvantage of the test is that it relies on subjective observation of the degree of fluorescence 
Evaluation of Two different Diagnostic Tests for Tropical Theileriosis

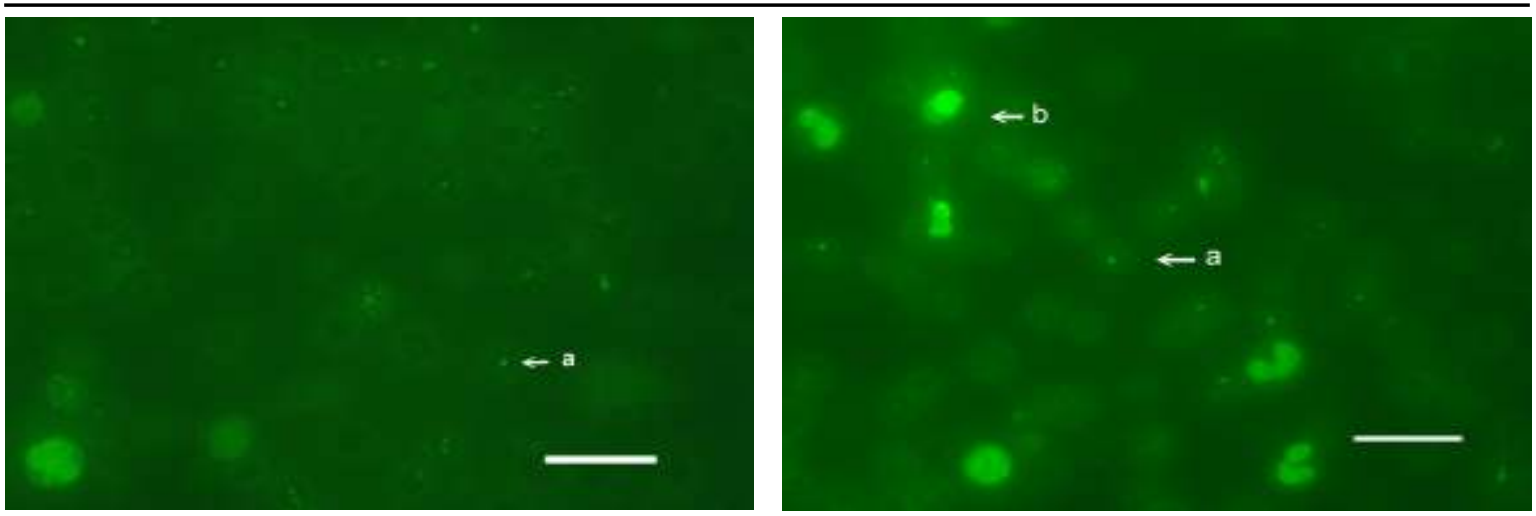

Figure. 1a \& 1b. A micrograph showing piroplasm of Theileria annulata in the RBCs (a) and merozoites or schizonts of Theileria annulata in the cytoplasm of a mononuclear cell (b) in acridine orange-stained blood smear (bar scale $=20 \mu \mathrm{m}$ ).

(Norval et al., 1992). Its inconvenience for the screening of large numbers of sera limits its use in epidemiological surveys (Kachani et al., 1992).

Fluorochromes have never been used for diagnosis of theileriosis. Hence, the present study was carried out to assess the applicability of acridine orange fluorescence technique as a rapid diagnostic test for tropical theileriosis. In this report we also describe the comparison of the Giemsa-stained thin blood film technique with the IFA test for diagnosis of tropical theileriosis

\section{Materials and methods}

Animals: The current study was carried out on 62 young ( $<1$ year) and 48 adult ( $>1$ year) Bos taurus Friesian cattle of both sexes suffering from clinical tropical theileriosis. The case definition was based on overt clinical signs (fever $>39^{\circ} \mathrm{C}$ and enlarged lymph nodes), detectable parasitaemia and parasitosis. These animals were presented to the Veterinary Teaching Hospital, College of Agriculture and Veterinary Medicine, Qassim University during the period from August 2006 to July 2008. For comparison, blood samples were also obtained from 25 young and 25 adult, clinically healthy, Friesian cattle, selected at random from different dairy farms in Qassim Region. Animals without overt clinical signs and carriers with very low parasitemia were not included.

Blood samples: Blood samples were collected by venipuncture into plain and EDTA-containing vacutainer tubes from adult and young Friesian cattle. Thin blood smears were prepared from the ear veins of all animals. Lymph node aspirates were prepared from suspected cases of tropical theileriosis.

Methods: The thin blood film and lymph node impression smears were stained with Giemsa. Thin blood smears of acridine orange were fixed with methanol and air dried. A drop of acridine orange stain ( $\mathrm{pH}$ 7.2) was placed on a clean cover slip, which was then placed on the film. Films were examined at 1000 magnification using a fluorescence microscope (Olympus BX51, Olympus Optical Co, Tokyo, Japan) fitted with a 100-Watt high pressure mercury burner and equipped with DP12 camera unit. All stained slides (Giemsa and fluorescence) were coded in a blind manner and examined by two independent microbiologists.

The piroplasm indirect fluorescent antibody test was carried out according to the method of Burridge and Kimber (1972) and OIE (2008). The conjugate, fluorescein isothiocyanate conjugated rabbit antibovine IgG (RAB-FITC), was obtained from Sigma (Sigma Chemical Company, USA). The reference positive serum was obtained from a chronically infected calf. The reference negative serum was obtained from a recently imported cow which was confirmed noninfected by blood smear and lymph node examination. The slides were examined as described above. Pilot studies showed disappearance of non-specific fluorescence at serum dilution level of $1 / 40$. This level was therefore, used as the starting dilution throughout the study.

\section{Results}

There was $100 \%$ correlation in the interpretation of slides stained with Giemsa and acridine orange, both with respect to positivity and negativity, between the two microbiologists. Piroplasms, schizonts and merozoites of Theileria in the cytoplasm of mononuclear cells and leukocytes fluoresce green (Fig. 1A, 1B).

Out of 110 young and adult Friesian cattle with 
Evaluation of Two different Diagnostic Tests for Tropical Theileriosis

Table-1. Prevalence of Theileria annulata antibodies in Friesian cattle in Qassim Region using the indirect fluorescent antibody technique.

\begin{tabular}{|c|c|c|c|c|c|c|}
\hline Groups & Blood smear Positive & IFA Positive & $\%$ & Blood smear negative & IFA Positive & $\%$ \\
\hline Calves & 62 & 53 & 85.5 & 25 & 13 & 52 \\
\hline Adults & 48 & 42 & 87.5 & 25 & 15 & 60 \\
\hline
\end{tabular}

Table 2. Comparison between results of the indirect fluorescent antibody technique and stained blood smear examination in diagnosis of Theileria annulata infection in Friesian cattle.

\begin{tabular}{|c|c|c|c|c|}
\hline \multirow[t]{2}{*}{ Smear } & & \multicolumn{3}{|c|}{ IFA test } \\
\hline & & Positive & Negative & Total \\
\hline Stained blood smear & $\begin{array}{c}\text { Positive } \\
\text { Negative } \\
\text { Total }\end{array}$ & $\begin{array}{l}83 \\
28 \\
111\end{array}$ & $\begin{array}{l}27 \\
22 \\
49\end{array}$ & $\begin{array}{c}110 \\
50 \\
160\end{array}$ \\
\hline
\end{tabular}

detectable parasitaemia $95(86.4 \%)$ were found positive by the indirect fluorescent antibody test. The seropositivity was higher in adults $(87.5 \%)$ than in young $(85.5 \%)$ (Table 1$)$. Of 50 examined known negative sera, 28 were found positive by the indirect fluorescent antibody test (Table 2). Using presence of T. annulata piroplasms as a gold standard, the IFA test has a sensitivity of $86.4 \%$ and specificity of $44 \%$.

\section{Discussion}

Theileria-infected young and adult exotic and cross-bred cattle suffered from severe and often fatal disease (Brown, 1990). Rapid detection of Theileria is a prerequisite for an effective treatment and thus in reducing the morbidity and mortality due to the disease. Giemsa-stained blood smear examination, which is the cornerstone in the laboratory diagnosis of Theileria, is time consuming, labour intensive and requires considerable expertise (Warhurst and Williams, 1996).

The results of the present study showed that the acridine orange stain correlated well with Giemsa stain with $100 \%$ sensitivity and specificity. Acridine orange could be used for detection of piroplasms of $T$. annulata in the RBCs and also for schizonts and/or merozoites of $T$. annulata in the cytoplasm of mononuclear cells.

The indirect fluorescent antibody test (IFAT) has been the most commonly used serum antibody assay for T. annulata and T. parva (Burridge, 1971; Burridge and Kimber, 1972; OIE, 2008). The clinical cases of $T$. annulata, which showed no serological responses, by the IFA could be in the early stages of infection before antibodies had been produced. Presence of $T$. annulata piroplasms was used as a gold standard to define an animal's true disease status. The sensitivity recorded for the IFA test $(87.5 \%)$ was comparable to earlier studies (Darghouth et al., 1996). The low specificity (44\%) recorded in the present investigation was obviously due to the falsehood of using absence of $T$. annulata. Piroplasms as a criterion (gold standard) for determining true negative animals. PCR technology for the detection of parasite DNA sequences in blood samples should be used as a gold standard instead of the insensitive blood smear examination technique.

These results indicate a relatively low sensitivity of the IFA test in the immunodiagnosis of acute $T$. annulata infections. These results came in line with earlier reports that the IFA test is not always sufficiently sensitive to detect all infected individuals (Darghouth et al., 1996). Further studies are needed to delineate the dynamics of antibody response during the course of $T$. annulata infections as the differences obtained could be due to different immunoglobulin class reacting or emerging at different intensity.

It is concluded that despite the requirement of expensive equipments acridine orange diagnostic technique is a valuable alternative for screening tropical theileriosis. The method may also have potential value in the diagnosis of Theileria parva, which causes East Coast fever and also for other Theileria species. On the other hand the IFA test was not found sufficiently sensitive and specific as has been reported earlier. The overall seroprevalence determined by the IFA in the present study was higher than expected in state of endemic instability as evidenced by the high disease incidence and case fatality rate. Results obtained showed that the IFA test is appropriate in epidemiological studies but not as complementary in the immunodiagnosis of acute $T$. annulata infections.

\section{Acknowledgments}

The author would like to thank Mr. Ibrahim 
Hassan of the Department of Microbiology, College of Medicine, Qassim University for technical assistance.

\section{References}

1. Brown, C.G.D (1990) Control of tropical theileriosis (Theileria annulata infection) of cattle. Parassitologia 32 23-31.

2. Burridge, M.J. (1971). Application of the indirect fluorescent antibody test for experimental East Coast fever Res. Vet. Sci. 12:338-341.

3. Burridge, M.J., Kimber, C.D. (1972). Duration of serological response to the indirect fluorescent antibody test for experimental East Coast fever (Theileria parva infection of cattle). Evaluation of a cell culture schizont antigen. Res.Vet. Sci. 13: 451-455.

4. Darghouth, M.E., Bouattour, A., Ben-Miled, L. and Sassi L. (1996). Diagnosis of Theileria annulata infection of cattle of Tunisia: Comparison of serology and blood smear. Vet. Res. 27: 613-621.

5. Dhar, S., Bhattacharyulu, Y., Gautam, O.P. (1973) Susceptibility of Indian water buffalo to Theileria annulata infection. Haryana Agricultural University Journal of Research 3: 27-31.

6. Dschunkowsky, E.B., Luhs, I. (1904). Die Piroplasmosen Rinder. Vorlaufäige Mitteilung. Zentralblatt für Bakteriologie, Parasitekunde, Infektions-krankheiten und Hygiene, Abteilung I35: 486-492.

7. Gill, B.S., Bhattacharyulu, Y. Kaur, D. (1977). Studies on the relationship between the quantum of infection and the ensuing reaction of cattle infected with Theileria annulata. Ann. Soc. Belg. Med. Trop. 57: 557-567.

8. Hashemi-Fesharki, R. (1991). Chemotherapeutic value of parvaquone and buparvaquone against Theileria annulata infection of cattle. Res. Vet. Sci. 50: 204-207.
9. Hänscheid, T. (1999). Diagnosis of malaria: a review of alternatives to conventional microscopy. Clin. Lab. Haematol. 21: 235-45.

10. Kachani, M., et.al.(1992). Stage-specific responses following infection with Theileria annulata as evaluated using ELISA. Parasitol. Res. 78: 43-47.

11. Keiser, J., Utzinger, J., Premji, Z., Yamagata, Y., Singer, B.H. (2002). Acridine Orange for malaria diagnosis: its diagnostic performance, its promotion and implementation in Tanzania, and the implications for malaria control. Ann. Trop. Med. Parasitol. 96: 643-654.

12. Kong, H.H., Chung, D.I. (1995). Comparison of acridine orange and Giemsa stains for malaria diagnosis. Korean. J. Parasitol. 33, 391-4.

13. Mehlhorn, H., Schein, E., Ahmed, J.S., (1994). Theileria. In: Parasitic Protozoa, Volume 7. (J.P. Kreier, ed.) Academic Press, Inc., pp. 269-271.

14. Norval, R.A.I., Perry, B.D., Young, A.S. (1992). The Epidemiology of Theileriosis in Africa. Academic Press, London, San Diego.

15. OIE (2008). Office International des Epizooties: Manual of Diagnostic Tests and Vaccines for Terrestrial Animals, $6^{\text {th }}$ edition.

16. Purnell, R.E. (1978). East Coast fever: some recent research in East Africa. Adv. Parasitol. 15: 83-132.

17. Robinson, P.M., 1982 Theileria annulata and its transmission - a review. Trop. Anim. Health Prod. 14: 3-13.

18. Uilenberg, G. (1981). Theilerial species of domestic livestock. In: Advances in the Control of Theileriosis: Proceedings of an International Conference Held at ILRAD, Nairobi, 9-13 February 1981. Editors, A.D. Irvin, M.P. Cunningham and A.S. Young, Martinus Nijhoff Publishers, The Hague, pp. 4-37.

19. Warhurst D.C., Williams, J.E. (1996). Laboratory Diagnosis of malaria. J. Clin. Pathol. 49: 533-538. 